\title{
Non-genetically Editing Dysbiotic Microbiome Using Probiotic-specific Prebiotics for Treatments of Human Skin Diseases
}

ISSN: 2639-0531

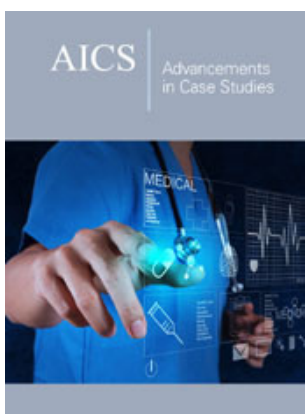

*Corresponding author: Jiang Y, America Diagnosis, Inc., USA

Submission: 眥 January 28, 2020

Published: 想 February 04, 2020

Volume 2 - Issue 3

How to cite this article: Huang RY, Raymond Herr D, Huang CM, Jiang Y. Nongenetically Editing Dysbiotic Microbiome Using Probiotic-specific Prebiotics for Treatments of Human Skin Diseases. Adv Case Stud.2(3). AICS.000538.2020. DOI: 10.31031/AICS.2020.02.000538

Copyright@ Yong J, This article is distributed under the terms of the Creative Commons Attribution 4.0 International License, which permits unrestricted use and redistribution provided that the original author and source are credited.

\author{
Huang RY ${ }^{1,2}$, Raymond Herr $\mathrm{D}^{3}$, Huang CM${ }^{4}$ and Jiang $\mathrm{Y}^{5 *}$ \\ ${ }^{1}$ Department of Mechanical and Aerospace Engineering, USA \\ ${ }^{2}$ Canyon Crest Academy, USA \\ ${ }^{3}$ Department of Pharmacology, Singapore \\ ${ }^{4}$ Department of Biomedical Sciences and Engineering, Taiwan \\ ${ }^{5}$ America Diagnosis, Inc., USA
}

\begin{abstract}
External interruption in the balance of microbes in the microbiome can lead to a dysbiotic microbiome which has been linked to a variety of human diseases. Antibiotics have been widely used for treatments of infectious diseases in humans, but they are generally not pathogen specific and run a risk of yielding antibiotic-resistant microbes. Inappropriate use of antibiotics may disrupt the homeostasis of human microbiome, causing dysbiotic microbiome and abolishing natural and self-curative of human body. Here, we review a new approach by using bacterial specific prebiotics to amplify the fermentation activity of probiotic bacteria against pathogens and keep the microbiome balanced for optimal health. The pros and cons of using skin prebiotic as a new modality for improvement of skin health are compared to other treatments including antibiotics, vaccines, bacteriophages, genetically modified/engineering bacteria and live skin probiotic bacteria.
\end{abstract}

Keywords: Dysbiotic; Prebiotic; Probiotic; Microbiome; Skin

\section{Introduction}

\section{Gene editing and genetically modified/engineering bacteria}

An uprising method to create advanced and specialized organisms is gene editing [1]. This process involves changing an organism's DNA by adding or removing a portion of their genome. Gene editing has been used in a variety of instances. One is in genetically modified organisms (GMOs) as commonly seen in plants. GMOs are created by introducing foreign genetic materials, forming a transgenic organism. Hence, by doing so, the organism's performance is enhanced for the desired outcome. For instance, a portion of fruits' genomes are edited to rot slower for the desired outcome of a longer shelf life. Another form of gene editing is the lately discovered clusters of regularly interspaced short palindromic repeats (CRISPR), specifically CRISPR associated with the Cas9 protein, discovered in a genome editing mechanism in bacteria. CRISPR-Cas9 works through using a guide RNA (gRNA) to help guide the system to bind to a specific region in the DNA and direct the Cas9 enzyme to cleave this sequence. This method is a promising weapon to combat cancer by genetically modifying T Cells to target specific proteins such as New York Esophageal Squamous Cell Carcinoma-1 (NY-ESO-1) on cancer cells [2]. To add on, it is through this system that scientists are seeking to edit the genetic material of bacteria to combat numerous diseases. Yet, there are ethical and scientific issues with gene editing for bacteria. In genetically editing bacteria, there is a risk that bacteria could pass the genes they carry to other bacteria or host cells, with unpredictable consequences [3]. When bacteria are transferred with foreign genes, they may develop resistance to current drugs, making human diseases harder to treat. Thus, genetically modifying/engineering bacteria may not be a practical means of treating or preventing diseases before positive results are shown from clinical trials. 


\section{Live beneficial or probiotic bacteria}

Without gene modification or engineering, many microbes naturally provide benefits to their hosts. For example, beneficial bacteria produce antimicrobial agents against pathogens [4] and probiotic bacteria undergo fermentation to help hosts to fight diseases [5] (Figure 1). Dysbiosis refers to a state with microbial imbalances within the human microbiome [6]. Yogurt is the best illustration of using gut probiotic bacteria to rebalance the gut dysbiosis. Reports show that short-chain fatty acids (SCFAs) produced by probiotic bacteria have been detected in infected tissues in humans [7-9]. Many SCFAs including acetic acid, butyric acid or succinic acid possess the decent antimicrobial activities against human pathogens. Furthermore, SCFA such as butyric acid can diminish inflammation via inhibition of histone deacetylase (HDAC) [10], suggesting the dual antimicrobial and anti-inflammatory activities of SCFAs. Unlike vaccines, the action of probiotic bacteria for treatments of infections does not directly call for host immune activation and may have little or no disruption to other commensal bacteria. In the current market, probiotic yogurts or supplements can be given by oral administration in humans. It has been shown that oral or topical probiotics can influence the host microbiome and may offer health benefits for patients [11]. Approaches using probiotics for the treatments of acne vulgaris and atopic dermatitis have been tested in vitro [12]. However, the Food and Drug Administration (FDA) does not yet approval live probiotic bacteria used for topical application onto human skin. One of concerns may include that these live probiotic bacteria may enter the bloodstream when they are directly inoculated onto skin with damaged skin barrier.

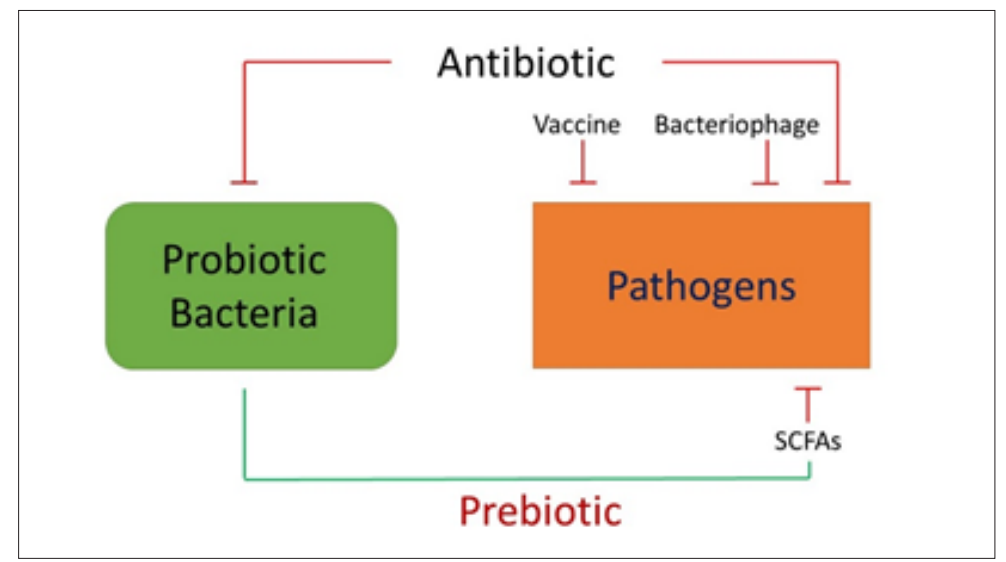

Figure 1: Probiotic bacteria fermentatively metabolize prebiotic to produce short-chain fatty acids (SCFAs) against pathogens. Development of bacteria specific prebiotic can avoid the some drawbacks of using antibiotic, vaccine, bacteriophage, or SCFAs for suppression of the growth of pathogens

The use of SCFAs in fermentation metabolites as therapeutic agents for treatments of human diseases may be an alternative for live probiotic bacteria. However, two pharmacokinetic drawbacks of SCFAs as antimicrobials and/or therapeutics are related to their rapid metabolization and failure to achieve concentrations in vivo [13]. SCFAs act on host cells through either inhibition of HDAC or activation of free fatty acid receptors [Ffar1; also known as G-protein coupled receptor 41 (GPR41) and Ffar2; GPR43) [14]. Literature has demonstrated that GW9508 (GlaxoSmithKline), an arylalkyl derivative of the propionic acid, activated the Ffar1 receptor, attenuated chemokine induction in keratinocytes and reduced cutaneous immune inflammation [15]. To conquer the problems of SCFAs with shorter half-lives, several SCFA analogs have been recently synthesized as antimicrobials against pathogens or anti-inflammatory agents via inhibition of HDAC [5,16]. Some SCFA analogs as HDAC inhibitors with anti-proliferative and inflammatory activities are in preclinical and clinical development, including pivaloylomethyl butyrate (AN-9), a pro-drug of butyric acid [17]. Although SCFA analogs may hold the potential as novel antimicrobial agents, their bacterial specificities have not been scientifically evaluated.

\section{Risks of antibiotics, vaccines and bacteriophages}

Lack of microbial specificity and development of resistance are primary problems in antibiotic therapy. Antibiotics without bacterial specificity for treatments of skin diseases may destroy the beneficial or probiotic bacteria that help rein in the over-growth of pathogens and maintain homeostasis of skin microbiome. Vaccines, that have been proven effective to prevent many human diseases [18,19]. However, vaccines, although microbial specific, highly relies on the immune status of humans. Phage therapy is as one of precision microbiome editing strategies that scientists take advantage of bacteriophages to specifically kill pathogens without interrupting other bacteria in host [20]. Although bacteriophages could be highly pathogen specific, many drawbacks of phage therapy have been reported [21]. For example, for selection of bacteriophages as potential safe antimicrobial organisms, detailed comprehensive features of genome and phenotypic properties may be necessary since bacteriophages hold negative characteristics such as lysogeny-associated genes, toxin or enzyme encoding genes [22]. In addition, scientists have not yet entirely appreciated the safety issue of phage therapy as many identified genes of 
bacteriophages express hypothetical and putative proteins with predicted or unknown functions. Additional downsides of phage therapy include that bacteriophages may transfer their genes from a microbe to another. It has been reported that bacteriophages can mediate the mechanism for genetic transduction [23] to integrate genes coding for virulence factors or antibiotic resistance [24] into hosts.

\section{Prebiotic approaches}

"Precision microbiome editing" can be delineated as a term for tailoring treatments of infections specifically targeting a species of probiotic, not other bacteria in the human microbiome. The precision microbiome editing approaches using prebiotic can exclusively trigger the fermentation of probiotic bacteria against pathogens. The approach has been demonstrated effective for treatments of skin diseases caused by the dysbiotic microbiome [25-27]. Different bacterial species make different enzymes that ferment specific sugar substrates as carbon sources. Staphylococcus epidermidis (S. epidermidis), Staphylococcus aureus (S. aureus) and Cutibacterium acnes (C. acnes; formerly Propionibacterium acnes), three main bacteria in the human skin microbiome, can ferment glucose to SCFAs [28-31]. However, S. epidermidis, but not S. aureus, can ferment mannose and galactose [30,31]. Previous results have showed that both $S$. epidermidis and C. acnes, but not $S$. aureus (USA300), can fermentatively metabolize glycerol [32]. Although S. aureus bacteria utilize several carbohydrates as substrates for fermentation, they cannot ferment ducitol (also called galactitol) and saccharic acid [31]. The ability of $S$. epidermidis and $C$. acnes to ferment ducitol and saccharic acid is undetermined. It has been documented that $S$. epidermidis can use sucrose as a carbon source to undergo fermentation and produce succinic acid to suppress the both in vitro and in vivo growth of $C$. acnes [26], a bacterium highly associated with dysbiotic acne vulgaris. S. epidermidis, but not $C$. acnes, can ferment sucrose to SCFAs.

Carbon atoms, as sources of bacterial fermentation, are tetravalent, allowing the formation of long carbon chains. There are four major categories for classification of carbon-based macromolecules in living things: carbohydrates, lipids, proteins, and nucleic acids. Each type of carbon-based molecules is composed of specific subunit molecules as monomers or multiple monomers as polymers. Polyethylene glycol (PEG) with long chains of carbon atoms is a synthetic water-soluble polymer. PEG polymers are greatly biocompatible and biodegradable molecules which have been approved by the U.S. FDA for transdermal drug delivery in humans [33]. PEG is nontoxic and approved by the FDA for use as excipients or as a carrier in different pharmaceutical formulations, foods, and cosmetics. PEG-derived polymers have been employed as a carbon source for bacterial fermentation. Scientists have reported that bacteria can secrete extracellular enzymes to depolymerize long chain PEGs [34]. Data suggested that PEG was taken up into the bacteria and subsequently degraded inside [35]. The depolymerization of PEG can be catalyzed after a modification of the terminal ethylene glycol (EG) unit by bacterial hydrolysis
[35]. The PEG fermentation of bacteria yielded acetaldehyde as an intermediate, which was further disproportionated to acetate and ethanol. It has been also reported that the propionate-forming bacteria can ferment PEG to acetate and propionate [36]. A methoxy poly(ethylene glycol)-b-poly( $\varepsilon$-caprolactone) (mPEG-PCL) copolymer functioned as a prebiotic of Staphylococcus lugdunensis, selectively triggering the bacterial fermentation for production of acetic and isovaleric acids against Candida parapsilosis [37], one of fungi in human dandruff flakes. S. epidermidis fermented the PEG dimethacrylate to SCFAs which can suppress the growth of $S$. aureus in the wound skin in mice [25]. Results from previous publication above clearly demonstrate the prebiotic property of PEG polymers for skin probiotic bacteria.

Inulin, a soluble plant fiber [38], and human milk oligosaccharides (HMOs) [39] have been used as prebiotic in the formula of probiotic yogurts or supplements for improvement of diseases caused by the dysbiotic gut microbiome. Plant extracts have been also verified as prebiotics for gut bacteria. Researchers found that turmeric extract not only was metabolized by gut probiotic bacteria including Lactobacillus rhamnosus GG (LGG) and Bifidobacterium animal is BB12, but also enhanced the growth of these bacteria [40]. To our knowledge, there are no prebiotics used for skin probiotic bacteria in the market. PEG di-methacrylate was demonstrated as a prebiotic for fermentation of skin S. epidermidis bacteria [25]. Several derivatives of PEG di-methacrylate PEG polymers with extremely low acute toxicity have been generally recognized as Safe (GRAS) by FDA. They may be an alternative of bacteriophages for editing skin microbiome [41] since they are relatively safer. Many derivatives of PEG di-methacrylate have been listed in International Nomenclature of Cosmetic Ingredients (INCI), revealing their potential as skin prebiotics.

\section{Limitations of using skin prebiotics}

Application of live probiotic bacteria onto skin has been not approved by FDA. Furthermore, the number of probiotic bacteria on human skin varies among individuals. Thus, the therapeutic effects of topically applied prebiotics to enhance the growth of skin probiotic bacteria likely vary among individuals. Acne treatment is one such example, as application of sufficient doses of prebiotics onto acne lesions specifically for $S$. epidermidis fermentation may be able to efficiently suppress the growth of $C$. acnes and $C$. acnes induced inflammation. However, since each individual acne patient may carry different numbers of probiotic $S$. epidermidis in an acne lesion. Thus, it will be hard for dermatologists to determine how many prebiotics should be topically applied onto acne lesions.

The normal skin surface exerts self-sterilizing activity which has been ascribed to various factors such as low $\mathrm{pH}$ or some antimicrobial agents including SCFAs. A number of SCFAs, although concentrations are relatively low, are commonly detected in the skin and in the secretions of skin glands, such as the sweat. Human sweat contained $0.1 \%$ lactic, $0.04 \%$ citric, $0.0096 \%$ acetic and $0.0062 \%$ propionic acid [42]. It is still not unknown how 
much SCFA can be produced by fermentation of skin probiotic bacteria. The concentration of total SCFAs in humans is $\sim 50$ $100 \mu \mathrm{M}$ in peripheral blood [43]. However, it has been reported that SCFAs locally produced by intestinal microbes in the human colon can reach a high level $(20-140 \mathrm{mM})$ [44] that can effectively kill local pathogens. Previous results [25] have shown that PEG di-methacrylate fermentation of $S$. epidermidis produced SCFAs including acetic, isovaleric, iso-butyric and propionic acids. The values of minimum bactericidal concentration (MBC) (reduction of at least one $\log _{10}$ C. acnes CFU) of SCFAs for C. acnes are in the range of 5 to $10 \mathrm{mM}$ [26]. These SCFAs can completely killed $C$. acnes in vitro at a concentration $>10 \mathrm{mM}$. It is not clear whether topical application of prebiotics can induce fermentation of skin probiotic bacteria to produce SCFAs at amounts in the $\mathrm{mM}$ range locally to hinder the growth of pathogens.

It has been documented that lipopolysaccharides (LPS) and peptidoglycans (PGN) found on the outer membrane gram -negative and -positive bacteria, respectively, exert toxic effects on human cells $[45,46]$. Literature has described that oral supplements of gram-negative probiotics such as mutaflor ${ }^{\circledR}$ and Symbioflor $2^{\circledR}$ are fairly safe, since they produce low amounts of LPS exclusively in gut, which will not increase the toxic dose of LPS in bloodstream [47]. However, it should be of concern as to whether administration of prebiotic will lead to a tremendous proliferation of probiotic bacteria, causing an increase in the amount of LPS or PGN in the body. Thus, when prebiotics are administered, LPS or PGN levels produced in humans may need to be determined in the future.

\section{Conclusion}

Bacterial interference via fermentation has been widely employed in the development of probiotic therapy. If the limitations of using skin prebiotics above can be overcome, topical application of prebiotics on the skin to rebalance the microbial dysbiosis in the skin microbiome not only creates a novel modality for promotion of skin health but also provides an alternative for using antibiotics, vaccines and bacteriophages for treatments of skin diseases.

\section{Acknowledgement}

We thank Damon Meyer, an assistant professor at College of Health Sciences, California, Northstate University, for providing the knowledge of bacterial cultures. We would also like to thank Dr. Renkun Chen, professor at Department of Mechanical and Aerospace Engineering, University of California, San Diego for offering a research internship as a student in Canyon Crest Academy.

This research did not receive any specific funding.

\section{References}

1. Lanza R, Russell DW, Nagy A (2019) Engineering universal cells that evade immune detection. Nat Rev Immunol 19(12): 723-733.

2. Mastaglio S, Genovese P, Magnani Z, Ruggiero E, Landoni E, et al. (2017) NY-ESO-1 TCR single edited stem and central memory T cells to treat multiple myeloma without graft-versus-host disease. Blood 130(5): 606-618.
3. Reardon S (2018) Genetically modified bacteria enlisted in fight against disease. Nature 558(7711): 497-498.

4. O Neill AM, Nakatsuji T, Hayachi A, Williams MR, Mills RH, et al. (2020) Identification of a human skin commensal bacterium that selectively kills cutibacterium acnes. J Invest Dermatol pii: S0022-202X (20)3004130045.

5. Traisaeng S, Herr DR, Kao HJ, Chuang TH, Huang CM (2019) A derivative of butyric acid, the fermentation metabolite of staphylococcus epidermidis, inhibits the growth of a staphylococcus aureus strain isolated from atopic dermatitis patients. Toxins (Basel)11(6): pii. E311.

6. DAS B, Nair GB (2019) Homeostasis and dysbiosis of the gut microbiome in health and disease. J Biosci 44(5): 117.

7. Menon S, Bharadwaj R, Chowdhary AS, Kaundinya DV, Palande DA (2007) Rapid identification of non-sporing anaerobes using nuclear magnetic resonance spectroscopy and an identification strategy. Indian J Med Microbiol 25(4): 330-335.

8. Demaerel P, Van Hecke P, Van Oostende S, Baert AL, Jaeken J, et al. (1994) Bacterial metabolism shown by magnetic resonance spectroscopy. Lancet 344(8931): 1234-1235.

9. Gorbach SL, Mayhew JW, Bartlett JG, Thadepalli H, Onderdonk AB (1976) Rapid diagnosis of anaerobic infections by direct gas-liquid chromatography of clinical speciments. J Clin Invest 57(2): 478-784.

10. Tong X, Yin L, Giardina C (2004) Butyrate suppresses cox-2 activation in colon cancer cells through HDAC inhibition. Biochem Biophys Res Commun 317(2): 463-471.

11.Yu Y, Dunaway S, Champer J, Kim J, Alikhan A (2020) Changing our microbiome: Probiotics in dermatology. Br J Dermatol 182(1): 39-46.

12. Mottin VHM, Suyenaga ES (2018) An approach on the potential use of probiotics in the treatment of skin conditions: Acne and atopic dermatitis. Int J Dermatol 57(12): 1425-1432.

13. Keshari S, Wang Y, Herr DR, Wang SM, Yang WC, et al. (2020) Skin cutibacterium acnes mediates fermentation to suppress the calcium phosphate-induced itching: A butyric acid derivative with potential for uremic pruritus. J Clin Med 9(2): e312.

14. Vinolo MA, Rodrigues HG, Nachbar RT, Curi R (2011) Regulation of inflammation by short chain fatty acids. Nutrients 3(10): 858-876.

15. Hara T, Hirasawa A, Ichimura A, Kimura I, Tsujimoto G (2011) Free fatty acid receptors FFAR1 and GPR120 as novel therapeutic targets for metabolic disorders. J Pharm Sci 100(9): 3594-3601.

16. Wang Y, Zhang L, Yu J, Huang S, Wang Z, et al. (2017) A co-drug of butyric acid derived from fermentation metabolites of the human skin microbiome stimulates adipogenic differentiation of adipose-derived stem cells: Implications in tissue augmentation. J Invest Dermatol 137(1): 46-56.

17. Hobdy E, Murren (2004) AN-9 (Titan). Curr Opin Investig Drugs 5(6): 628-634.

18. Nakatsuji T, Liu YT, Huang CP, Gallo RL, Huang CM (2008) Antibodies elicited by inactivated propionibacterium acnes-based vaccines exert protective immunity and attenuate the IL-8 production in human sebocytes: Relevance to therapy for acne vulgaris. J Invest Dermatol 128(10): 2451-2457.

19. Nakatsuji T, Liu YT, Huang CP, Gallo RL, Huang CM (2008) Vaccination targeting a surface sialidase of $p$. acnes: Implication for new treatment of acne vulgaris. Plos One 3(2): e1551.

20. Pincus NB, Reckhow JD, Saleem D, Jammeh ML, Datta SK, et al. (2015) Strain specific phage treatment for staphylococcus aureus infection is influenced by host immunity and site of infection. Plos One 10(4): e0124280.

21. Haq IU, Chaudhry WN, Akhtar MN, Andleeb S, Qadri I (2012) Bacteriophages and their implications on future biotechnology: A review. Virol J 9. 
22. Drulis-Kawa Z, Majkowska-Skrobek G, Maciejewska B, Delattre AS, Lavigne R (2012) Learning from bacteriophages - advantages and limitations of phage and phage-encoded protein applications. Current protein \& peptide science 13(8): 699-722.

23. Chen J, Quiles-Puchalt N, Chiang YN, Bacigalupe R, Fillol-Salom A, et al. (2018) Genome hypermobility by lateral transduction. Science 362(6411): 207-212.

24. Martins IJ (2017) Antibiotic resistance involves antimicrobial inactivation in global communities. SAJ Pharma Pharmacol 4: 101.

25. Kao MS, Huang S, Chang WL, Hsieh MF, Huang CJ, et al. (2017) Microbiome precision editing: Using PEG as a selective fermentation initiator against methicillin-resistant staphylococcus aureus. Biotechnol J 12(4).

26. Wang Y, Kao MS, Yu J, Huang S, Marito S, et al. (2016) A precision microbiome approach using sucrose for selective augmentation of staphylococcus epidermidis fermentation against propionibacterium acnes. Int J Mol Sci 17(11): E1870.

27. Lolou V, Panayiotidis MI (2019) Functional role of probiotics and prebiotics on skin health and disease. Fermentation 5(2): 41.

28. Sivakanesan R, Dawes EA (1980) Anaerobic glucose and serine metabolism in staphylococcus epidermidis. J Gen Microbiol 118(1): 143157.

29. Barbirato F, Chedaille D, Bories A (1940) Propionic acid fermentation from glycerol: Comparison with conventional substrates. Appl Microbio Biotech 47(4): 441-446.

30. Robbins GB, Lewis KH (1940) Fermentation of sugar acids by bacteria. Bacteriol 39(4): 399-404.

31. Safonova TB, Shcherbakova NA, Afanaseva TI, Sobolev VR (1978) Importance of carbohydrate tests for interspecies differentiation of staphylococci. Zh Mikrobiol Epidemiol Immuno Biol 9: 98-101.

32. Yang JJ, Chang TW, Jiang Y, Kao HJ, Chiou BH, et al. (2018) Commensal staphylococcus aureus provokes immunity to protect against skin infection of methicillin-resistant staphylococcus aureus. Int J Mol Sci 19(5): E1290.

33. Che J, Okeke CI, Hu ZB, Xu J (2015) DSPE-PEG: A distinctive component in drug delivery system. Curr Pharm Des 21(12): 1598-1605.

34. Tokiwa Y, Suzuki T (1983) Hydrolysis of polyesters by lipases. Nature 270: 76-78.
35. Schink B, Stieb M (1983) Fermentative degradation of polyethylene glycol by a strictly anaerobic, gram-negative, nonsporeforming bacterium, Pelobacter venetianus sp. nov. Appl Environ Microbiol 45(6): 1905-1913.

36. Oda Y, Oida N, Urakami T, Tonomura K (1997) Polycaprolactone depolymerase produced by the bacterium Alcaligenes faecalis. FEMS Microbiol Lett 152(2): 339-343.

37. Kao MS, Wang Y, Marito S, Huang S, Lin WZ, et al. (2016) The mPEGPCL copolymer for selective fermentation of Staphylococcus lugdunensis against candida parapsilosis in the human microbiome. J Microb Biochem Technol 8(4): 259-265.

38. Akram W, Garud N, Joshi R (2019) Role of inulin as prebiotics on inflammatory bowel disease. Drug Discov Ther 13(1): 1-8.

39. Moukarzel S, Bode L (2017) Human milk oligosaccharides and the preterm infant: A Journey in sickness and in health. Clin Perinatal 44(1): 193-207.

40. Ghiamati YF, Soleimanian ZS, Vanden WE, Folkerts G (2019) Turmeric extract: Potential use as a prebiotic and anti-inflammatory compound? Plant Foods Hum Nutr 74(3): 293-299.

41. Liu J, Yan R, Zhong Q, Ngo S, Bangayan NJ, et al. (2015) The diversity and host interactions of propionibacterium acnes bacteriophages on human skin. ISME J 9(9): 2078-2093.

42. Burtenshaw JM (1942) The mechanism of self-disinfection of the human skin and its appendages. J Hyg (Lond) 42(2): 184-210.

43. Cummings JH, Pomare EW, Branch WJ, Naylor CP, Macfarlane GT (1987) Short chain fatty acids in human large intestine, portal, hepatic and venous blood. Gut 28(10): 1221-1227.

44. Garland SH (2011) Short chain fatty acids may elicit an innate immune response from preadipocytes: A potential link between bacterial infection and inflammatory diseases. Med Hypotheses 76(6): 881-883.

45. Martins IJ (2018) Bacterial lipopolysaccharides and neuron toxicity in neurodegenerative diseases. Neurol Neurosurg 1(1): 1-3.

46. Chartier CMP, Kulakauskas S (2014) Cell wall structure and function in lactic acid bacteria. Microb Cell Fact 13(Suppl 1): S9.

47. Wassenaar TM, Zimmermann K (2018) Lipopolysaccharides in food, food supplements, and probiotics: Should we be worried? Eur J Microbiol Immunol (Bp) 8(3): 63-69. 